\title{
Analysis of Oral Microbiota Revealed High Abundance of Prevotella Intermedia in Gout Patients
}

\author{
Juan Liu ${ }^{\mathrm{a}}$ Li Cui ${ }^{\mathrm{b}} \quad$ Xinmin Yan ${ }^{\mathrm{a}} \quad$ Xinyuan Zhao ${ }^{\mathrm{c}}$ Jinluo Cheng ${ }^{\mathrm{a}}$ Lei Zhou ${ }^{\mathrm{a}}$ \\ Jianbo $\mathrm{Gao}^{\mathrm{a}}$ Zhong $\mathrm{Cao}^{\mathrm{b}}$ Xinhua Ye ${ }^{\mathrm{a}}$ Shen $\mathrm{Hu}^{\mathrm{b}}$ \\ ${ }^{a}$ Changzhou Second People's Hospital, Nanjing Medical University, Changzhou, China, 'bchool of \\ Dentistry, University of California, Los Angeles, USA, 'Department of Endodontics, Stomatological \\ Hospital, Southern Medical University, Guangzhou, China
}

\section{Key words}

Gout - Hyperuricemia - 16S rDNA sequencing - Oral microbiota • Prevotella intermedia • Streptococcus anginosus $\bullet$ Serratia marcescens

\begin{abstract}
Background/Aims: Microbes reside in a number of body sites, including the oral cavity, and are associated with the progression of many systemic diseases. In this study, we aimed to investigate the effects of gout and hyperuricemia (HUA) on the composition of oral microbiomes. Methods: Analysis of the oral microbiota from 12 gout patients, 11 HUA patients, and 19 healthy control subjects was performed using a deep sequencing approach, and validation of significant changes in Prevotella intermedia and Serratia marcescens in new patient cohorts was performed using quantitative PCR (qPCR). Results: Our analysis indicated that both gout and HUA significantly altered the composition of the oral microbiome in patients. Patients with gout or HUA had significantly greater levels of salivary Prevotella intermedia but significantly lower levels of Serratia marcescens than healthy control subjects. Conclusion: We demonstrated the association between the oral microbiome and gout and HUA for the first time. In particular, $16 \mathrm{~S}$ sequencing and QPCR analysis revealed significantly higher levels of oral Prevotella intermedia in gout/HUA patients, which suggests that these patients might be at risk for the development of periodontitis.
\end{abstract}

(C) 2018 The Author(s)

Published by S. Karger AG, Basel

\section{Introduction}

Gout is a systemic disease characterized by the deposition of monosodium urate crystals in the joints and surrounding tissues [1,2]. The prevalence and incidence of gout have risen in recent years around the world. Asymptomatic hyperuricemia (HUA) is considered an

J. Liu and L. Cui contributed equally to this work.

Xinhua Ye

and Shen $\mathrm{Hu}$ 
important risk factor for the development of gout. Both gout and HUA have been associated with a number of pathological conditions such as metabolic syndrome, obesity, hypertension, stroke, cardiovascular diseases, and renal diseases [3-6]. However, the effects of gout/HUA on oral health remain unknown.

In recent years, saliva has attracted widespread interest as a means of simple, repeatable, and rapid disease testing. The advantages of using saliva for disease diagnostics and prognostics include ease of access, noninvasive sample collection, low cost, and reduced risk of infectious disease transmission [7, 8]. In fact, the composition of the salivary microbiota has been closely linked to multiple systemic diseases such as cardiovascular diseases and diabetes mellitus, as well as preterm delivery of low birth weight infants [9-11]. Most salivary bacteria are derived from the oral cavity, although some may also originate in the esophagus and the upper respiratory tract [12]. The survival and growth of bacteria in the oral cavity are dependent on the oral environment. The levels of salivary urates significantly increase in gout/HUA [13, 14], which implies that the $\mathrm{pH}$ might be lower in the oral cavity of gout/HUA patients. Therefore, gout/HUA may change the composition of the oral microbiome and lead to pathological conditions in the oral cavity.

We investigated the global variations in salivary microbiota among patients with gout/ HUA. We quantified the composition of bacterial flora in saliva samples obtained from 12 gout patients, 11 HUA patients, and 19 healthy controls by $16 \mathrm{~S}$ rDNA sequencing using the Illumina HiSeq 2000 sequencer. This approach can identify more than 500 prevalent human bacterial species, with up to 100,000 bacterial sequences per sample. This comprehensive and highly sensitive analysis provides a new approach to investigating the relationship between gout/HUA and oral microbial composition. Data analysis was then performed to identify bacteria at the species level that showed significant differences among the three study groups. Quantitative PCR (qPCR) was subsequently used to confirm the changes among several selected bacteria of interest. Prevotella intermedia is an oral bacterium frequently associated with periodontal diseases [15]. We found that it was significantly more abundant in saliva samples from gout and HUA patients than those from healthy controls. Our findings demonstrated that patients with gout/HUA not only have significantly altered oral microbiomes versus healthy people, but also might be prone to periodontitis.

\section{Materials and Methods}

\section{Sample collection}

The study complied with the Helsinki Declaration and was approved by the Medical Ethics Committee at the Changzhou Second People's Hospital, Nanjing Medical University. All saliva samples were collected at the Changzhou Second People's Hospital according to the approved protocol. Written informed consent was obtained from all participants. Since smoking may affect the growth of bacteria in the oral cavity, only subjects with a non-smoking history were selected. Saliva samples were collected from the gout/ HUA patients before treatment. None of the patients or control subjects manifested any pathological conditions such as diabetes, cardiovascular diseases, immune dysfunction, herpes viral infections, or oral diseases (periodontitis, oral mucosal ulcers, etc.). Unstimulated whole saliva was harvested from patients and healthy controls according to our standardized protocol for sample collection [8]. Tables 1-2 list the clinicopathological parameters of the patients. The enrolled subjects in the discovery cohort (sequencing analysis) were completely independent from those in the validation cohort (qPCR analysis).

\section{Sequencing analysis of bacterial flora of saliva samples}

The DNA content in saliva samples was isolated by sodium dodecyl sulfate lysis, following phenol extraction to remove proteins from the saliva. Recovered DNA from saliva samples was amplified using qPCR, as previously described [16]. The amplified samples were sequenced using an Illumina HiSeq 2000, and V3 and V6 of 16S rDNAs were analyzed. To ensure high quality reads, we adopted stringent conditions to process the sample sequences. In brief, the following processing steps were performed: 1) all reads were assigned to corresponding samples by allowing one mismatch to the sample barcode and two mismatches 


\section{Cellular Physiology Cell Physiol Biochem 2018;49:1804-1812 \begin{tabular}{l|l|l} 
and Biochemistry 10.1159/000493626 & $\begin{array}{l}\text { C } 2018 \text { The Author(s). Published by S. Karger AG, Basel } \\
\text { www.karger.com/cpb }\end{array}$ \\
\hline
\end{tabular}}

Liu et al.: Gout and Oral Microbiome

to the adjacent PCR primer; 2) the reads were subsequently de-noised via the PyroNoise algorithm; 3) reads containing ambiguous nucleotides or a homopolymer longer than 8 base pairs (bp) were removed, as were sequences shorter than 200 bp or longer than 1000 bp; 4) the reads were aligned using a nearest alignment space terminationbased sequence aligner to a custom reference based on the SILVA alignment, and sequences that did not align to the anticipated region of the reference alignment were discarded; 5) chimeric sequences identified by the UCHIME algorithm were removed; and 6) the reads were classified using a Bayesian classifier with the Ribosomal Database Project. Sequences of mitochondria or unknowns (i.e., those reads that could not be classified at the kingdom level) were removed. Finally, all effective reads were clustered into operational taxonomic units (OTUs) based on $97 \%$ sequence similarity, using the mothur program (version 1.35.1). Taxonomic profiling of samples at different taxonomy levels (phylum, class, order, family, genus, and species) were created using QIIME software (version 1) [12].

\section{$q P C R$}

Candidate bacterial biomarkers identified from sequencing analysis were further confirmed by qPCR on a new set of gout/HUA patients and control subjects. Specific PCR primers for the detection of $16 \mathrm{~S}$ rDNAs were designed and are listed in Table 3.

\section{Statistical analysis}

Data analysis was performed with GraphPad Prism and MedCalc software. Group differences were evaluated by one-way analysis of variance. Statistical significance in this study was determined by $P$ values and a $P$ value less than 0.05 was considered statistically significant.
Table 1. Clinicopathological parameters of the enrolled subjects for the deep sequence analysis

\begin{tabular}{lccc}
\hline Clinical parameters & \multicolumn{3}{c}{ Group (Mean \pm SD) } \\
& Gout $(\mathrm{n}=12)$ & HUA (n=11) & CTRL ( $\mathrm{n}=19)$ \\
\hline Age (yr) & $54.42 \pm 12.87$ & $53.50 \pm 10.92$ & $55.95 \pm 9.50$ \\
Gender (male) & $8 / 12$ & $9 / 11$ & $15 / 19$ \\
BMI (Kg/m2) & $24.76 \pm 3.25$ & $24.88 \pm 1.16$ & $22.46 \pm 1.36$ \\
Systolic pressure (mm Hg) & $130.17 \pm 11.14$ & $128.75 \pm 8.20$ & $124.37 \pm 8.99$ \\
Diastolic pressure (mm Hg) & $79.00 \pm 4.18$ & $77.75 \pm 3.74$ & $76.73 \pm 6.33$ \\
Fasting Blood glucose (mmol/L) & $5.92 \pm 1.00$ & $5.38 \pm 0.71$ & $5.14 \pm 0.45$ \\
Serum uric acid ( $\mu \mathrm{mol} / \mathrm{L})$ & $690.12 \pm 180.81$ & $523.18 \pm 82.55$ & $263.01 \pm 70.43$ \\
Total cholesterol $(\mathrm{mmol} / \mathrm{L})$ & $4.74 \pm 1.03$ & $4.60 \pm 0.57$ & $4.44 \pm 0.84$ \\
Blood urea nitrogen $(\mathrm{mmol} / \mathrm{L})$ & $6.03 \pm 1.20$ & $5.36 \pm 1.08$ & $5.14 \pm 1.15$ \\
Serum creatinine $(\mu \mathrm{mol} / \mathrm{L})$ & $99.43 \pm 21.80$ & $88.11 \pm 12.05$ & $64.59 \pm 18.51$ \\
\hline
\end{tabular}

Table 2. Clinicopathological parameters of the enrolled subjects for the qPCR validation study

\begin{tabular}{lccc}
\hline Clinical parameters & \multicolumn{3}{c}{ Group (Mean \pm SD) } \\
& Gout $(\mathrm{n}=30)$ & HUA $(\mathrm{n}=30)$ & CTRL $(\mathrm{n}=30)$ \\
\hline Age (yr) & $52.20 \pm 14.33$ & $54.17 \pm 7.60$ & $55.77 \pm 10.17$ \\
Gender (male) & $24 / 30$ & $25 / 30$ & $24 / 30$ \\
BMI (Kg/m2) & $25.45 \pm 1.90$ & $25.25 \pm 1.76$ & $22.90 \pm 0.77$ \\
Systolic pressure $(\mathrm{mm} \mathrm{Hg})$ & $128.86 \pm 11.31$ & $130.50 \pm 11.50$ & $125.65 \pm 6.92$ \\
Diastolic pressure $(\mathrm{mm} \mathrm{Hg})$ & $79.87 \pm 5.76$ & $80.13 \pm 7.28$ & $77.19 \pm 5.61$ \\
Fasting Blood glucose $(\mathrm{mmol} / \mathrm{L})$ & $5.91 \pm 0.72$ & $5.41 \pm 0.84$ & $5.31 \pm 0.63$ \\
Serum uric acid $(\mu \mathrm{mol} / \mathrm{L})$ & $713.30 \pm 199.50$ & $476.00 \pm 105.90$ & $229.10 \pm 79.60$ \\
Total cholesterol $(\mathrm{mmol} / \mathrm{L})$ & $5.05 \pm 1.20$ & $4.43 \pm 0.65$ & $4.40 \pm 0.74$ \\
Blood urea nitrogen $(\mathrm{mmol} / \mathrm{L})$ & $6.36 \pm 1.72$ & $5.50 \pm 0.93$ & $5.36 \pm 1.32$ \\
Serum creatinine $(\mu \mathrm{mol} / \mathrm{L})$ & $95.57 \pm 18.60$ & $85.41 \pm 10.89$ & $62.05 \pm 15.51$
\end{tabular}

Table 3. The primers used for qPCR analysis of $16 \mathrm{~S}$ rDNA

\begin{tabular}{lc}
\hline Name of bacterial species & Primer sequence \\
\hline Prevotella intermedia & F: CCACATATGGCATCTGACGTG \\
R: TCAATCTGCACGCTACTTGG \\
Ferratia marcescens & F: GGTGAGCTTAATACGTTCATCAATTC \\
Streptococcus anginosus & F: GTTTTGCAGAAGCGATTGTC \\
\end{tabular}




\section{Cellular Physiology Cell Physiol Biochem 2018;49:1804-1812 \begin{tabular}{ll|l} 
and Biochemistry Published online: 20 September, 2018 & $\begin{array}{l}\text { C) } 2018 \text { The Author(s). Published by S. Karger AG, Basel } \\
\text { www.karger.com/cpb }\end{array}$ \\
\hline
\end{tabular}

\section{Results}

The similarities and differences in the taxonomic compositions of oral microbiota among gout, HUA, and control subjects

High quality paired-end reads were combined to tags when two paired-end reads overlapped; a consensus sequence was generated by fast length adjustment of short reads. The criteria for the combination of tags were as follows: 1) minimal overlapping length, $15 \mathrm{bp}$; 2) mismatching ratio of overlapped region, $\leq 0.1$; and 3) paired-end reads without overlap were removed. A total of 1, 305, 698 tags were obtained from all samples, with an average of 31, $088 \pm 326$ tags for each sample. The average length of each tag was $252 \pm 1$ bp. The total numbers of read pairs, tag numbers, and connected tag numbers in each group are summarized in Fig. 1.

Tags were clustered into OTUs with a 97\% threshold. The similarities and differences in the taxonomic compositions of saliva microbiomes were visualized using a Venn diagram. The results showed that most OTUs $(n=332)$ were shared among all three groups. However, 39 OTUs were shared only between gout patients and control subjects, 21 OTUs were shared only between HUA patients and healthy controls, and 11 OTUs were shared only between gout and HUA patients. In addition, all three individual groups had unique OTUs (Fig. 2).

Fig. 1. Basic sample information among different groups. The total pairs read number, tag number and connected tag number in the participants from each study group (gout, HUA and healthy control).

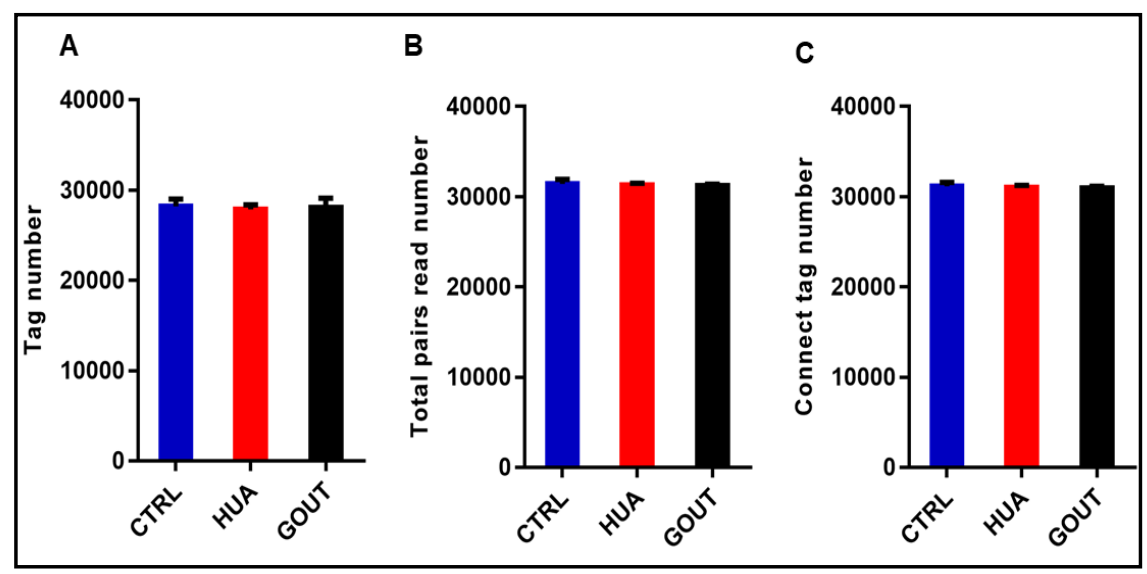

Fig. 2. The OTUs among different groups. Most OTUs $(n=332) \quad$ were shared among the gout, HUA and healthy control groups. However, thirty-nine OTUs were shared only between gout patients and healthy control subjects. Twenty-one OTUs were shared only

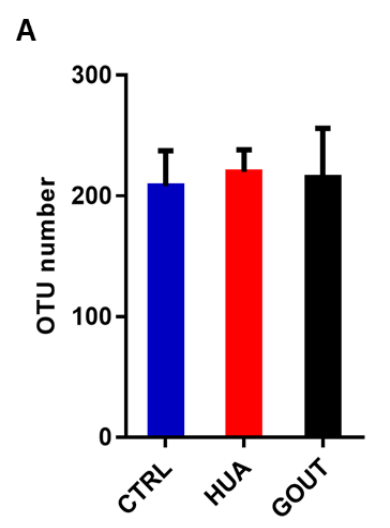

B

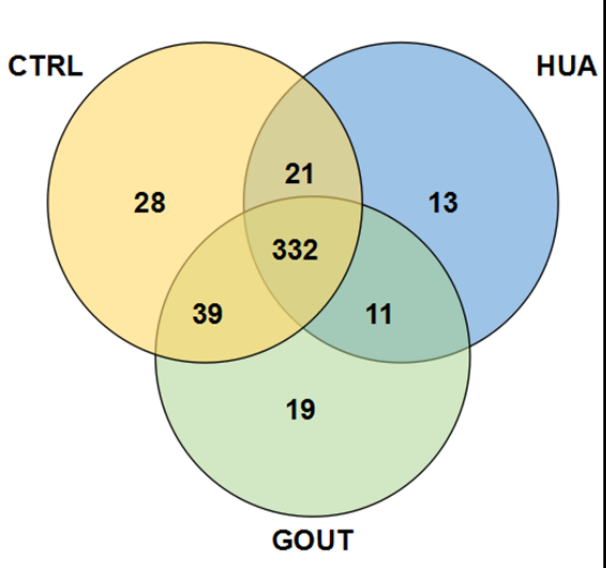

between HUA

patients and healthy controls and 11 OTUs were only shared between gout and HUA patients. 


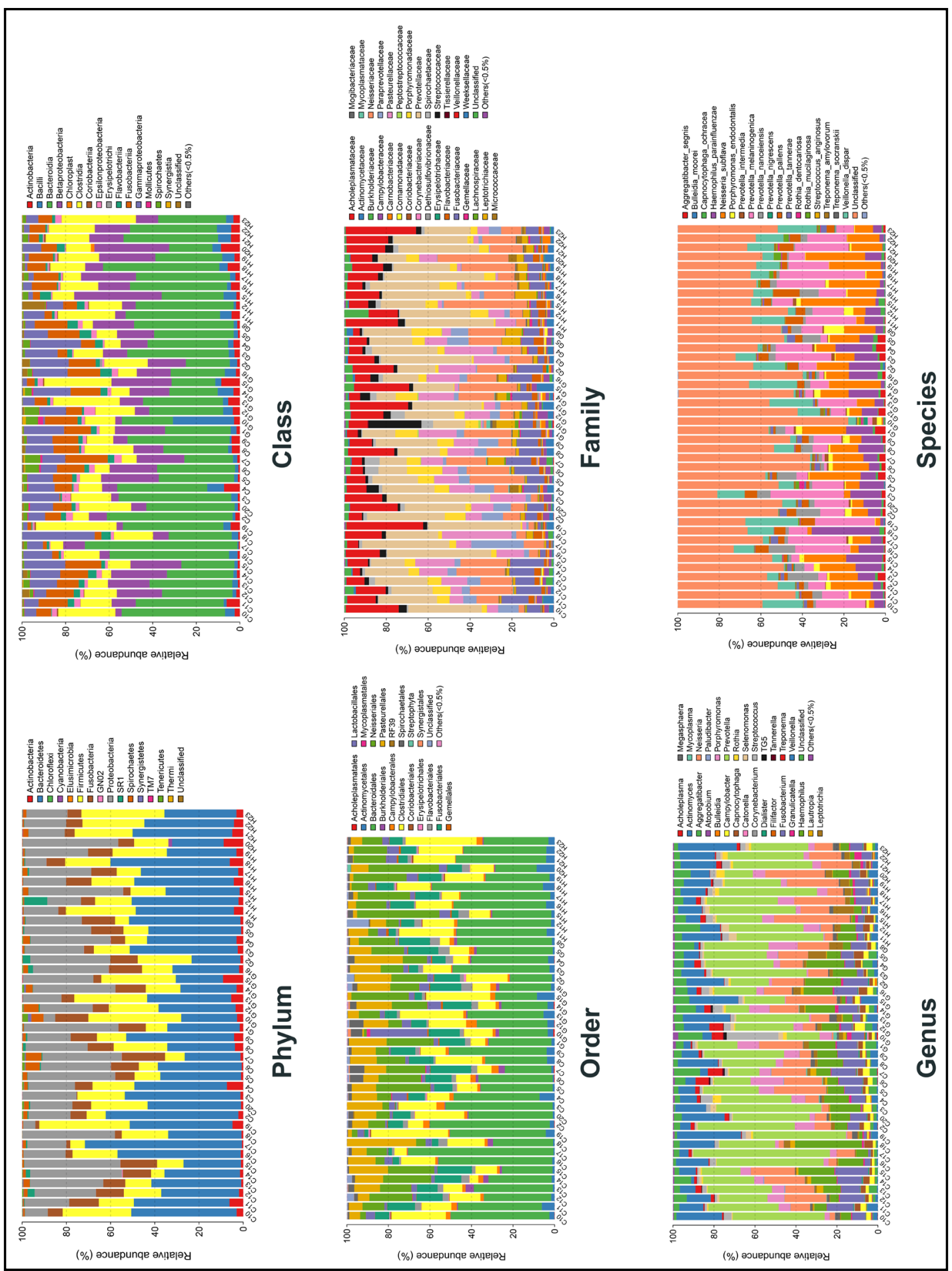

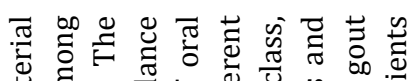

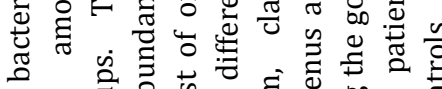

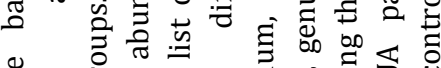

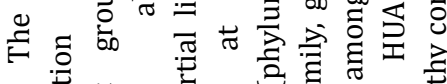

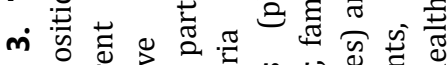

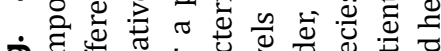

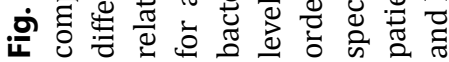
KARGER 
Gout and HUA significantly altered the composition of oral microbiota in patients

We then analyzed the relative abundance of a partial list of oral bacteria among the gout, HUA, and control groups at different taxonomic levels (phylum, class, order, family, genus, and species). As shown in Fig. 3, sequencing analysis indicated that the composition of oral microbiomes differed significantly among the gout patients, HUA patients, and healthy control subjects. Table 4 lists the relative abundance of a partial list of oral bacteria at the species level between gout patients and healthy controls, and between HUA patients and healthy controls. Prevotella intermedia was found to be significantly more abundant in HUA patients and gout patients compared to healthy controls. Similarly, Streptococcus anginosus was significantly more abundant in both gout and HUA patients versus healthy controls. However, Serratia marcescens was detected in healthy controls only-not in gout or HUA patients.

Oral bacteria species were selectively abundant in gout and HUA

To validate the three potential bacterial markers (Prevotella intermedia, Streptococcus anginosus, and Serratia marcescens), we used qPCR to quantify their levels in new patients (gout/HUA) and control cohorts. The qPCR results indicated that the amounts of Prevotella intermedia were highest in gout patients, and lowest in healthy controls $(P<0.001)$. The amounts of Serratia marcescens were higher in saliva samples from healthy controls but significantly lower in both gout and HUA patients (Fig. 4). This confirmed the sequencing analysis results and supported the feasibility of using qPCR to quantify the levels of selected bacterial species.

Table 4. Relative abundance of a partial list of oral bacteria among gout patients, HUA patients and healthy controls based on sequencing analysis

\begin{tabular}{lcccc}
\hline \multirow{2}{*}{ Taxonomy/Species } & \multicolumn{2}{c}{ GOUT vs. CTRL } & \multicolumn{2}{c}{ HUA vs. CTRL } \\
& Fold change & P Value & Fold change & P Value \\
\hline Desulfomicrobium orale & 4.03 & 0.36 & Not detected in HUA & N/A \\
Serratia marcescens & Not detected in gout & N//A & Not detected in HUA & N/A \\
Streptococcus anginosus & 3.15 & 0.04 & 3.05 & 0.04 \\
Prevotella intermedia & 4.97 & 0.01 & 1.79 & 0.03 \\
Rothia mucilaginosa & 1.53 & 0.22 & 4.57 & 0.05 \\
Prevotella nanceiensis & 0.54 & 0.12 & 0.49 & 0.08 \\
Neisseria subflava & 1.18 & 0.45 & 1.59 & 0.09 \\
\hline
\end{tabular}

Fig. 4. Validation of selective oral bacteria. The level of Prevotella intermedia was at the highest in the patients with gout, while at the lowest in the healthy controls $\left({ }^{* * *} \mathrm{P}<0.001\right)$. Serratia marcescens was found to be higher in the saliva samples from healthy controls but it was barely

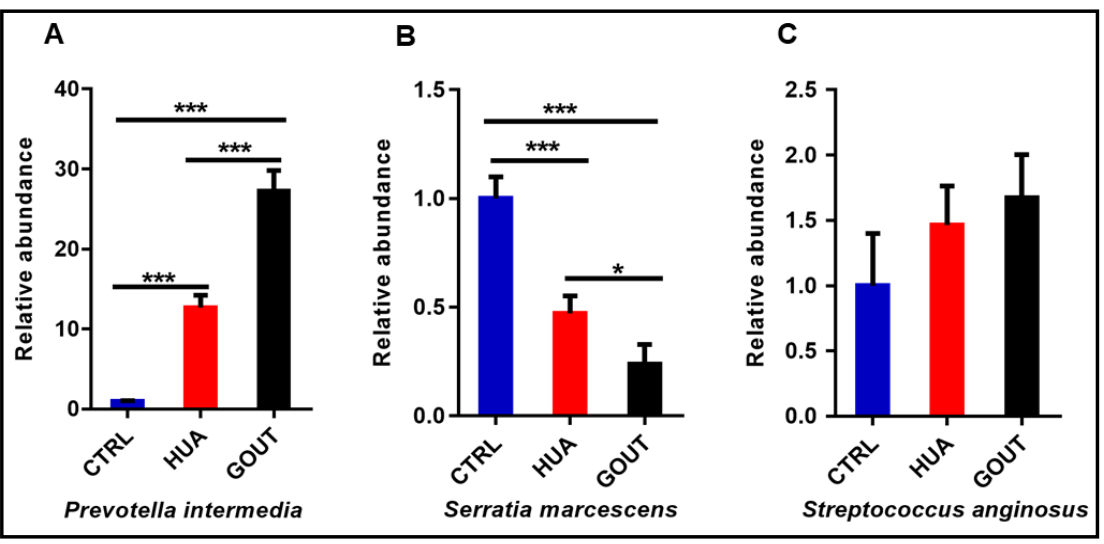
detectable in both gout and HUA patients $\left({ }^{*} \mathrm{P}<0.05,{ }^{* * *} \mathrm{P}<0.001\right)$. The comparison of the abundance of Streptococcus anginosus among the three groups did not show significance difference $(\mathrm{P}>0.05)$. 
Although a similar trend was observed for Streptococcus anginosus by qPCR analysis to sequencing analysis, the amounts of the bacterium among gout, HUA, and healthy control groups was not significantly different $(P>0.05)$.

\section{Discussion}

Our comprehensive sequencing analysis demonstrated that oral microbiota profiles were significantly altered in patients with gout or HUA compared with healthy control subjects. Detailed analysis of the deep sequencing data showed that the diversity and abundance of oral bacteria varied among gout patients, HUA patients, and healthy controls at multiple taxonomic levels. At the species level in particular, Prevotella intermedia and Streptococcus anginosus were found to be more abundant in both gout and HUA patients, whereas Serratia marcescens was less abundant in both gout and HUA patients than in healthy controls. Based on these results, we performed qPCR validation studies using new and independent patient/ control cohorts, and further confirmed significantly higher levels of Prevotella intermedia and lower levels of Streptococcus anginosus in gout and HUA patients than in healthy controls.

The intrinsic relationship between the oral microbiota and gout/HUA is currently unknown. The normal oral microbiota is composed of important resident microbes that play critical protective roles against colonization by extrinsic bacteria that could affect oral and systemic health. Indeed, previous studies have provided critical insights into how abnormal compositions of oral microbiota may be related to oral and systemic diseases [17-21]. On the other hand, a recent study also showed that systemic diseases have significant influence on the oral microbiota [22]. Salivary urate is reported to be significantly increased in gout and higher concentrations may change the oral microenvironment, such as the $\mathrm{pH}$ of saliva [13], which has been demonstrated to be an important factor that affects the balance of oral microbial communities [23,24]. In addition to uric acid, there might be many other metabolites that are altered in the saliva of gout/HUA patients that contribute to the modification of the normal oral microbiota. Our findings clearly showed that significant changes occur in the oral microbiomes of patients with gout or HUA. While it is unknown how the altered oral microbiota might affect the progression of gout disease, it is entirely possible that gout and HUA cause the altered composition of oral microbiota in these patients, which might lead to additional oral pathological conditions.

Periodontitis is defined as an inflammatory disease of tooth supporting tissues and is associated with colonization by different oral pathogens such as Porphyromonas gingivalis, Tannerella forsythia, and Treponema denticola. In fact, Prevotella intermedia is also an important periodontal pathogen that has been linked to inflammation of the gums and the progression of periodontitis. Many bacteria including Porphyromonas gingivalis grow only at neutral $\mathrm{pH}$, while Prevotella intermedia is able to grow under acidic conditions and proliferates by utilizing glucose [25]. It is possible that a relatively acidic oral microenvironment might facilitate the growth of Prevotella intermedia. Patients with gout have been observed to be at increased risk of hypertension, diabetes mellitus, metabolic syndrome, and kidney and cardiovascular diseases. However, the underlying reasons remain largely unknown. Our studies suggested evidence for one of the possible explanations. First, gout and HUA cause changes in the oral microbiota (e.g., Prevotella intermedia). This may lead to oral conditions such as periodontitis and could aggravate the progression of systemic diseases. Second, Prevotella intermedia or other oral pathogens might enter the circulatory system and have noticeable effects on certain immune cells, cytokine production, and effector molecule expression, thus inducing both innate and adaptive immune responses. This would not be surprising considering that numerous studies have proven that oral bacterial pathogens are associated with cardiovascular diseases and diabetes mellitus. However, further studies are certainly warranted to confirm the pathogenic role of Prevotella intermedia. 


\section{Cellular Physiology Cell Physiol Biochem 2018;49:1804-1812 and Biochemistry Published online: 20 September, $2018 \mid \begin{aligned} & \text { DO 2018 The Author(s). Published by S. Karger AG, Basel } \\ & \text { www.karger.com/cpb }\end{aligned}$ \\ Liu et al.: Gout and Oral Microbiome}

Serratia marcescens is an opportunistic pathogen that causes nosocomial infections. It is ubiquitous in animals and resistant to many traditional antibiotics. Interestingly, Serratia marcescens may produce a toxic substance that selectively kills cancer cell lines [26], which suggests this microorganism might also be conducive to health under normal conditions. Our results showed that Serratia marcescens is present in the saliva from healthy controls but barely detectable in patients with gout or HUA. However, the underlying reasons need further investigation. Streptococcus anginosus (previously known as Streptococcus milleri) is a group of streptococci species that are strongly virulent pathogens, even though they reside as part of the normal flora in the oral cavity. Members of Streptococcus anginosus have been isolated from dental caries and periodontal diseases [27, 28]. The presence of these organisms in the oral cavity predisposes individuals to oral diseases. Therefore, our findings showing that the levels of Streptococcus anginosus are higher in the saliva from gout and HUA patients further support the suggestion that gout and HUA cause changes in the normal oral microbial communities and may contribute to the pathogenesis of oral diseases.

To the best of our knowledge, this is the first study to reveal the potential relationship between gout/HUA and the oral microbiome. Our results clearly showed that patients with gout/HUA are predisposed to potentially pathological conditions in the oral cavity due to alterations in the oral microbiome. In addition, disturbances in the oral microbiota of gout/ HUA patients could be linked to specific systemic conditions. Thus, it is important for patients with gout/HUA to pay close attention to their oral health.

\section{Disclosure Statement}

The authors declare that no conflicts of interest exist.

\section{References}

-1 Kuo CF, Grainge MJ, Zhang W, Doherty M: Global epidemiology of gout: prevalence, incidence and risk factors. Nat Rev Rheumatol 2015;11:649-662.

-2 Pascual E, Addadi L, Andrés M, Sivera F: Mechanisms of crystal formation in gout-a structural approach. Nat Rev Rheumatol 2015;11:725-730.

-3 Nguyen UD, Zhang Y, Louie-Gao Q, Niu J, Felson DT, LaValley MP, Choi HK: Obesity paradox in recurrent attacks of gout in observational studies: clarification and remedy. Arthritis Care Res 2017;69:561-566.

-4 Krishnan E, Baker JF, Furst DE, Schumacher HR: Gout and the risk of acute myocardial infarction. Arthritis Rheum 2006;54:2688-2689.

5 Gibson TJ: Hypertension, its treatment, hyperuricaemia and gout. Curr Opin Rheumatol 2013;25:217-222.

-6 Yu KH, Kuo CF, Luo SF, See LC, Chou IJ, Chang HC, Chiou MJ: Risk of end-stage renal disease associated with gout: a nationwide population study. Arthritis Res Ther 2012;14:83.

7 Hu S, Loo JA, Wong DT: Human saliva proteome analysis and disease biomarker discovery. Expert Rev Proteomics 2007;4:531-538.

-8 Cui L, Elzakra N, Xu S, Xiao GG, Yang Y, Hu S: Investigation of three potential autoantibodies in Sjogren's syndrome and associated MALT lymphoma. Oncotarget 2017;8:30039-30049.

-9 Slocum C, Kramer C, Genco CA: Immune dysregulation mediated by the oral microbiome: potential link to chronic inflammation and atherosclerosis. J Intern Med 2016;280:114-128.

$>10$ Saini R, Saini S, Saini SR: Periodontitis: A risk for delivery of premature labor and low birth weight infants. J Nat Sci Biol Med 2011;2:50-52.

11 Mealey BL, Oates TW: American Academy of Periodontology: Diabetes mellitus and periodontal diseases. J Periodontol 2006;77:1289-1303.

12 Yan X, Yang M, Liu J, Gao R, Hu J, Li J, Zhang L, Shi Y, Guo H, Cheng J, Razi M, Pang S, Yu X, Hu S: Discovery and validation of potential bacterial biomarkers for lung cancer. Am J Cancer Res 2015;5:3111-3122.

13 Zhao J, Huang Y: Salivary urate as a noninvasive biomarker for monitoring the efficacy of urate-lowering therapy in a patient with chronic gouty arthropathy. Clin Chim Acta 2015;450:115-120. 


\section{Cellular Physiology Cell Physiol Biochem 2018;49:1804-1812 and Biochemistry \begin{tabular}{c|c|} 
DOI: 10.1159/000493626 \\
Published online;20 September, 2018
\end{tabular} \\ Liu et al.: Gout and Oral Microbiome}

14 Owen-Smith B, Quiney J, Read J:Salivary urate in gout, exercise, and diurnal variation. Lancet 1998;351:1932.

15 Kamma JJ, Nakou M, Manti FA:Microbiota of rapidly progressive periodontitis lesions in association with clinical parameters. J Periodontol 1994;65:1073-1078.

16 Paster BJ, Boches SK, Galvin JL, Ericson RE, Lau CN, Levanos VA, Sahasrabudhe A, Dewhirst FE: Bacterial diversity in human subgingival plaque. J Bacteriol 2001;183:3770-3783.

-17 Astafurov K, Elhawy E, Ren L, Dong CQ, Igboin C, Hyman L, Griffen A, Mittag T, Danias J: Oral microbiome link to neurodegeneration in glaucoma. PLoS One 2014;9:e104416.

-18 Chukkapalli SS, Easwaran M, Rivera-Kweh MF, Velsko IM, Ambadapadi S, Dai J, Larjava H, Lucas AR, Kesavalu L: Sequential colonization of periodontal pathogens in induction of periodontal disease and atherosclerosis in LDLRnull mice. Pathog Dis 2017;75:1.

19 Arweiler NB, Netuschil L: The Oral Microbiota. Adv Exp Med Biol 2016;902:45-60.

20 Wang F, Jiang Y, Huang X, Liu Q Zhang Y, Luo W, Zhang F, Zhou P, Lin J, Zhang H: Pro-inflammatory cytokine TNF- $\alpha$ attenuates BMP9-induced osteo/odontoblastic differentiation of the stem cells of dental apical papilla (SCAPs). Cell Physiol Biochem 2017;41:1725-1735.

-21 Imai H, Fujita T, Kajiya M, Ouhara K, Yoshimoto T, Matsuda S, Takeda K, Kurihara H. Mobilization of TLR4 into lipid rafts by aggregatibacter actinomycetemcomitans in gingival epithelial cells. Cell Physiol Biochem 2016;39:1777-1786.

22 Corrêa JD, Calderaro DC, Ferreira GA, Mendonça SM, Fernandes GR, Xiao E, Teixeira AL, Leys EJ, Graves DT, Silva TA: Subgingival microbiota dysbiosis in systemic lupus erythematosus: association with periodontal status. Microbiome 2017;5:34.

23 Zhou J, Jiang N, Wang Z, Li L, Zhang J, Ma R, Nie H, Li Z: Influences of pH and iron concentration on the salivary microbiome in individual humans with and without caries. Appl Environ Microbiol 2017;83:e02412-e02416.

24 Takahashi N, Schachtele CF: Effect of pH on the growth and proteolytic activity of Porphyromonas gingivalis and Bacteroides intermedius. J Dent Res 1990;69:1266-1269.

25 Takahashi N, Saito K, Schachtele CF, Yamada T: Acid tolerance and acid-neutralizing activity of Porphyromonas gingivalis, Prevotella intermedia and Fusobacterium nucleatum. Oral Microbiol Immunol 1997;12:323-328.

26 Deorukhkar AA, Chander R, Ghosh SB, Sainis KB: Identification of a red-pigmented bacterium producing a potent anti-tumor N-alkylated prodigiosin as Serratia marcescens. Res Microbiol 2007;158:399-404.

27 Drucker DB, Green RM: Dental caries induced by Streptococcus milleri. J Dent Res 1977;56:1062.

28 Rawlinson A, Duerden BI, Goodwin L: New findings on the microbial flora associated with adult periodontitis. J Dent 1993;21:179-184. 